\title{
Spectrally encoded confocal microscopy for diagnosing breast cancer in excision and margin specimens
}

\author{
Elena F Brachtel ${ }^{1}$, Nicole B Johnson², Amelia E Huck ${ }^{1}$, Travis L Rice-Stitt ${ }^{1}$, Mark G Vangel ${ }^{3,4}$, Barbara L Smith ${ }^{5}$, \\ Guillermo J Tearney ${ }^{1,6,7}$ and Dongkyun Kang ${ }^{6}$
}

\begin{abstract}
A large percentage of breast cancer patients treated with breast conserving surgery need to undergo multiple surgeries due to positive margins found during post-operative margin assessment. Carcinomas could be removed completely during the initial surgery and additional surgery avoided if positive margins can be determined intraoperatively. Spectrally encoded confocal microscopy (SECM) is a high-speed reflectance confocal microscopy technology that has a potential to rapidly image the entire surgical margin at subcellular resolution and accurately determine margin status intraoperatively. In this study, in order to test the feasibility of using SECM for intraoperative margin assessment, we have evaluated the diagnostic accuracy of SECM for detecting various types of breast cancers. Forty-six surgically removed breast specimens were imaged with an SECM system. Side-by-side comparison between SECM and histologic images showed that SECM images can visualize key histomorphologic patterns of normal/benign and malignant breast tissues. Small $(500 \mu \mathrm{m} \times 500 \mu \mathrm{m})$ spatially registered SECM and histologic images ( $n=124$ for each) were diagnosed independently by three pathologists with expertise in breast pathology. Diagnostic accuracy of SECM for determining malignant tissues was high, average sensitivity of 0.91 , specificity of 0.93 , positive predictive value of 0.95 , and negative predictive value of 0.87 . Intra-observer agreement and inter-observer agreement for SECM were also high, 0.87 and 0.84 , respectively. Results from this study suggest that SECM may be developed into an intraoperative margin assessment tool for guiding breast cancer excisions.
\end{abstract}

Laboratory Investigation (2016) 96, 459-467; doi:10.1038/labinvest.2015.158; published online 18 January 2016

Breast cancer excision followed by radiation therapy is a standard breast-conserving therapy for early-stage invasive breast cancers. During the breast excision procedure, primary tumor is surgically removed with a rim of uninvolved breast tissue to allow for negative margins. ${ }^{1}$ Although breast excision generally provides a better cosmetic outcome and causes less morbidity than mastectomy, it carries a risk of leaving residual tumor behind in the patient, which can cause local recurrence of the breast cancer. An unacceptably large percentage of lumpectomy patients, $\sim 20-40 \%$, are found to have positive margins on post-operative histologic assessment of the surgical specimen..$^{2-4}$ These patients are required to undergo additional surgeries, which pose unnecessary physical and psychological burdens on the patients and an economic burden on the health care system. A few intraoperative margin assessment methods including frozen section analysis ${ }^{5,6}$ and touch preparation analysis ${ }^{7,8}$ are used in some institutions and have been shown to reduce the need for additional surgeries. However, these methods are not widely used, partly because of technical limitations, sampling bias, and the requirement of specialist interpretation.

Optical imaging technologies hold great promises for intraoperative margin assessment applications, as they can examine optical properties and/or histomorphologic features of freshly excised tissues without the need for frozen section or other tissue preparations. Optical spectroscopy technologies have previously been evaluated for determining margin status intraoperatively. Reflectance spectroscopy measures scattering and absorption properties of freshly excised breast tissues, which can be used to differentiate between benign and malignant patterns. Several studies of imaging breast specimens with reflectance spectroscopy showed that it can detect

\footnotetext{
Department of Pathology, Massachusetts General Hospital, Boston, MA, USA: ${ }^{2}$ Department of Pathology, Beth Israel Deaconess Medical Center, Boston, MA, USA: ${ }^{3}$ Department of Radiology, Massachusetts General Hospital, Boston, MA, USA; ${ }^{4}$ Biostatistics Center, Massachusetts General Hospital, Boston, MA, USA; ${ }^{5}$ Gillette Center for Women's Cancers and Department of Surgery, Massachusetts General Hospital, Boston, MA, USA; ${ }^{6}$ Wellman Center for Photomedicine, Massachusetts General Hospital, Boston, MA, USA and ${ }^{7}$ Harvard-MIT Division of Health Sciences and Technology, Boston, MA, USA 
positive margins with high accuracy. ${ }^{9-14}$ Raman spectroscopy can obtain quantitative chemical information of breast tissue, which can be used to distinguish cancerous tissues from normal/benign tissues. Previous studies have shown that Raman spectroscopy can provide high diagnostic accuracy for detecting malignant breast tissues. ${ }^{15,16}$

Optical microscopy technologies have higher resolution than aforementioned optical spectroscopy technologies and might have an advantage in detecting small or infiltrating tumors. Optical coherence tomography (OCT) visualizes architectural differences between normal and malignant tissues, and has been shown to determine margin status with high accuracy. ${ }^{17}$ OCT technology with cellular resolution, termed optical coherence microscopy, has also been shown to accurately determine margin status. ${ }^{18}$ Fluorescence confocal microscopy in conjunction with topical administration of $0.01 \%$ proflavine can visualize nuclear details of breast tissues and has been found to accurately diagnose neoplastic tissues. ${ }^{19}$ A nonlinear microscopy approach, where two-photon microscopy is used to visualize nuclei and second harmonic generation microscopy is used to visualize collagens, can generate images that appear similar to histologic images and provide high diagnostic accuracy. ${ }^{20}$ Reflectance confocal microcopy (RCM), when used with topical administration of $5 \%$ acetic or citric acid, has been shown to visualize nuclear features of various breast tissue types. ${ }^{21,22}$ Although these optical microscopy technologies show a potential capability for accurately determining margin status intraoperatively, imaging speed needs to be sufficiently high, to examine the entire surgical margin within a short procedural time and subsequently provide comprehensive margin information.

Spectrally encoded confocal microscopy (SECM) is a highspeed RCM technology that has a potential to rapidly image the entire surgical specimen at subcellular resolution. SECM uses a diffraction grating to encode a line field of view with wavelength. ${ }^{23}$ The spectral encoding enables line imaging of the tissue without using any beam scanning mechanism and therefore can increase the imaging speed of RCM significantly. Using a high-speed wavelength-swept source (repetition rate $=100 \mathrm{kHz}$ ) and a fast detector unit, SECM was previously demonstrated to rapidly image a human esophageal tissue over an area of $1 \mathrm{~cm}^{2}$ in $15 \mathrm{~s}^{24}$ When used during breast cancer surgery, the high speed of SECM could enable the surface of an entire surgical specimen to be rapidly imaged. SECM images can then be used to visually evaluate the margin status. Although SECM is expected to visualize key histomorphologic features associated with normal/benign and malignant breast tissues as previous RCM studies have shown, its diagnostic accuracy needs to be confirmed before it can be used for clinical intraoperative applications.

In this study, we evaluated the SECM diagnostic accuracy in determining malignant breast tissues. Surgically removed breast specimens were imaged with an SECM system. SECM images were compared with corresponding histologic images to identify histomorphologic features visualized in SECM

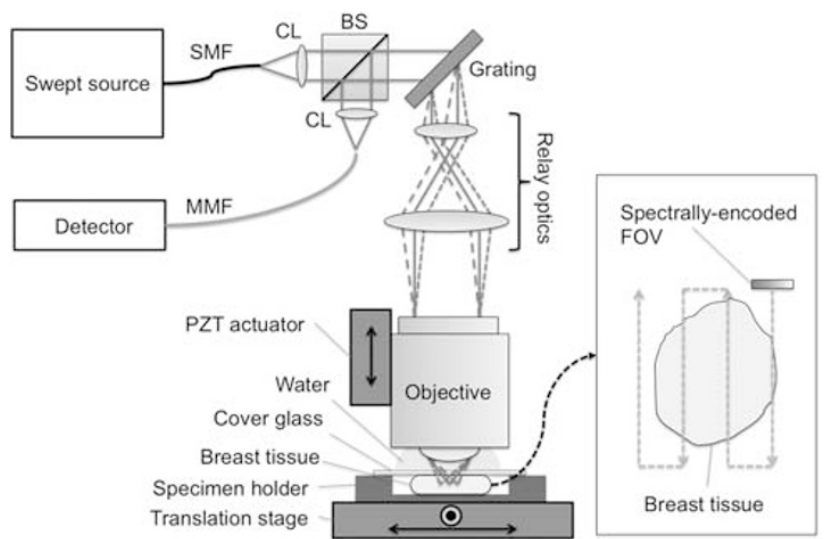

Figure 1 Schematic of SECM system. BS, beam splitter; CL, collimation lens; FOV, field of viw; MMF, multi-mode fiber; $\mathrm{PZT}$, piezo-electric transducer; SECM, spectrally encoded confocal microscopy; SMF, single-mode fiber.

images. Diagnostic accuracy of SECM was evaluated in comparison with the gold standard histologic diagnoses. Intra- and inter-observer agreements were also evaluated.

\section{MATERIALS AND METHODS SECM System}

We have used an SECM system that was previously developed for imaging gastroesophageal biopsy tissues. ${ }^{25,26} \mathrm{~A}$ schematic of the SECM system is shown in Figure 1. Light from a custom wavelength-swept source (repetition rate $=5 \mathrm{kHz}$; central wavelength $=1320 \mathrm{~nm}$; bandwidth $=70 \mathrm{~nm}$ ) was collimated by a collimation lens (OZ optics; $f=11 \mathrm{~mm}$ ) and diffracted by a transmission grating (Wasatch Photonics; groove density $=1100$ lines $/ \mathrm{mm}$ ). Diffracted light was delivered to the objective lens by relay optics (magnification $=2.4$ ). A high-NA objective lens (UPlanApo/IR $\times 60$, Olympus; $\mathrm{NA}=1.2$; water immersion) was used to focus the light on the tissue. Light reflected from the breast tissue was collected by the objective lens and travelled back to the beam splitter. Half of the light was reflected at the beam splitter and coupled into a multi-mode fiber and then to a photo detector. The SECM system had lateral resolution of $1.3 \mu \mathrm{m}$ and axial resolution of $2.4 \mu \mathrm{m}$. The width of the spectrally encoded line was $115 \mu \mathrm{m}$. Large-area SECM images of the tissue were acquired by raster scanning the tissue relative to the spectrally encoded line using a two-axis motorized translation stage (Nanomover 11NCM001, Melles Griot). Imaging depth was changed by translating the objective lens with a piezoelectric transducer actuator (8302, New Port).

\section{Breast Tissue Imaging}

We acquired and imaged breast tissues under a Partners Healthcare IRB-approved protocol (Protocol 2011P001417). Breast tissues were obtained from the Massachusetts General Hospital (MGH) surgical pathology laboratory directly after the tissues were removed from the patients. After gross examination and diagnostic sampling of a mastectomy or a 
breast excision specimen, selected fresh breast tissue fragments $($ size $=\sim 0.5-2 \mathrm{~cm}$ ) were sampled and transferred to the SECM system. Breast tissues were de-identified and each tissue was assigned with a unique study number. A total of 46 samples were obtained from 35 surgical specimens.

Each breast tissue was treated with $5 \%$ acetic acid, to enhance nuclear contrast. ${ }^{21,22}$ The breast tissue was then placed onto the translation stage underneath the objective lens. Large-area SECM images (size $=4 \times 2 \mathrm{~mm}^{2}-$ $10 \times 4.8 \mathrm{~mm}^{2}$ ) were obtained from several focal planes (number of focal planes $=2-10$ ) covering imaging depth range of $0-200 \mu \mathrm{m}$. SECM imaging time ranged from 10 to 15 min. Once SECM imaging was completed, the tissue was then prepared as hematoxylin and eosin (H\&E)-stained histologic slides per standard procedure. The tissue was cut en face to achieve good spatial registration between SECM and histologic images. Histologic slides were scanned by a whole slide scanner (Nanozoomer, Hamamatsu; $\times 20$ objective lens with NA of 0.75 ; scanning resolution $=0.46 \mu \mathrm{m}$ ). Digital images of the histologic slides were used for image analysis.

\section{Evaluation of Diagnostic Accuracy}

SECM and histologic images obtained from the same breast tissue were compared at various magnifications (Figure 2). SECM images were displayed with an inverted color map, which made highly scattering features, eg, cell nuclei appear dark and surrounding tissue bright. An image reader (DK) who was blinded to the histologic diagnoses reviewed multiple SECM images obtained from different focal planes and determined the focal plane that had highest spatial correlation to the histologic image. The image reader then identified small regions (size of each region $=500 \mu \mathrm{m} \times 500 \mu \mathrm{m}$ ) that exhibited similar microscopic features between SECM and histologic images, and saved the SECM and histologic regions as uncompressed TIFF and JPEG image files, respectively. We used JPEG file type for histologic images, as the whole slide images were saved with JPEG compression. Each JPEG image was displayed as a $15.9 \mathrm{~cm} \times 15.9 \mathrm{~cm}^{2}(700$ pixels $\times 700$ pixels $)$ on a 13.3" screen (Mac Book Pro, Apple). At this display condition, there was no noticeable image degradation. A total of 154 pairs of registered SECM and histologic images were generated.

The 154 histologic images were then reviewed by a senior breast pathologist (EFB), who made diagnoses on each histologic image into either one of the six normal/benign categories (adipose, fibrous, ducts/glands, inflammation, proliferative, and unspecified normal/benign) or one of the seven malignant categories (invasive ductal carcinoma (IDC), invasive lobular carcinoma (ILC), unspecified invasive carcinoma (IC), ductal carcinoma in situ (DCIS), lobular carcinoma in situ, unspecified carcinoma in situ, and unspecified malignant). Diagnoses by the senior pathologist were used as the gold standard during the diagnostic accuracy evaluation. After completing the gold standard diagnoses, the senior pathologist reviewed SECM images in comparison with corresponding histologic images, to identify histomorphologic features visualized in SECM images.

Three pathologists with expertise in breast pathology (NBJ, $\mathrm{AEH}$, and TLRS), who were blinded to the prior diagnoses by the senior pathologist, were trained to read SECM images. During the training session, each pathologist was presented with 30 pairs of SECM and histologic images side by side along with a written summary of histomorphologic features visualized in SECM images. After the training session, each pathologist reviewed 124 SECM images and prospectively rendered diagnosis for each SECM image as one of the six normal/benign or one of the seven malignant tissue categories listed above. The three pathologists also made diagnoses on 124 corresponding histologic images. Each pathologist conducted two sessions of SECM image review and two sessions of histologic image review to determine intraobserver agreement. In each image-review session, images were presented in a random order. There was at least a $24-\mathrm{h}$ washout period between image review sessions. Sensitivity and specificity for determining malignant tissues for each pathologist and each image review session were evaluated in comparison with the gold standard. Positive predictive value $(\mathrm{PPV})$, negative predictive value (NPV), and accuracy were also calculated. Intra-observer agreement was evaluated by calculating Cohen's $\kappa$ for each pathologist and each modality. Inter-observer agreement was calculated by pairwise comparison, where Cohen's $\kappa$ was calculated between two pathologists' diagnoses. Six $\kappa$-values were generated for each of the SECM and histologic diagnoses. Two-tailed $P$-values were calculated to compare sensitivity, specificity, intra-observer agreement, and inter-observer agreement measurements between SECM and histologic diagnoses. Logistic regression was used to calculate overall difference in sensitivity and specificity between SECM and histology.

\section{RESULTS}

\section{SECM Image of Normal/Benign Breast Tissue}

In SECM images, the structures appear on a gray scale in black and white, whereas histologic images stained by $\mathrm{H} \& \mathrm{E}$ show the cytoplasm and collagenous tissue as pink colour and the nuclei as blue colour. Representative SECM and histologic images of normal/benign breast tissues are shown in Figure 3. In an SECM image of fibrous tissue (Figure 3a), collagen of the fibrous tissue appears as a dark wavy structure that is similar to that of the corresponding histologic image (Figure 3b). Adipose cells (asterisks) are visualized as bright circles in an SECM image of the fat tissue (Figure 3c). The fat cells also exhibit crystalline-appearing structures centrally. An SECM image of the benign ducts and glands (Figure 3e) shows several regularly shaped glands. Each gland is clearly delineated by cell nuclei (arrows in inset, Figure 3e) that are similar in appearance to the nuclei seen in the corresponding histologic image (Figure 3f). Both SECM and histologic images of inflamed tissue (Figures $3 \mathrm{~g}$ and $\mathrm{h}$ ) show numerous lymphocytes. 

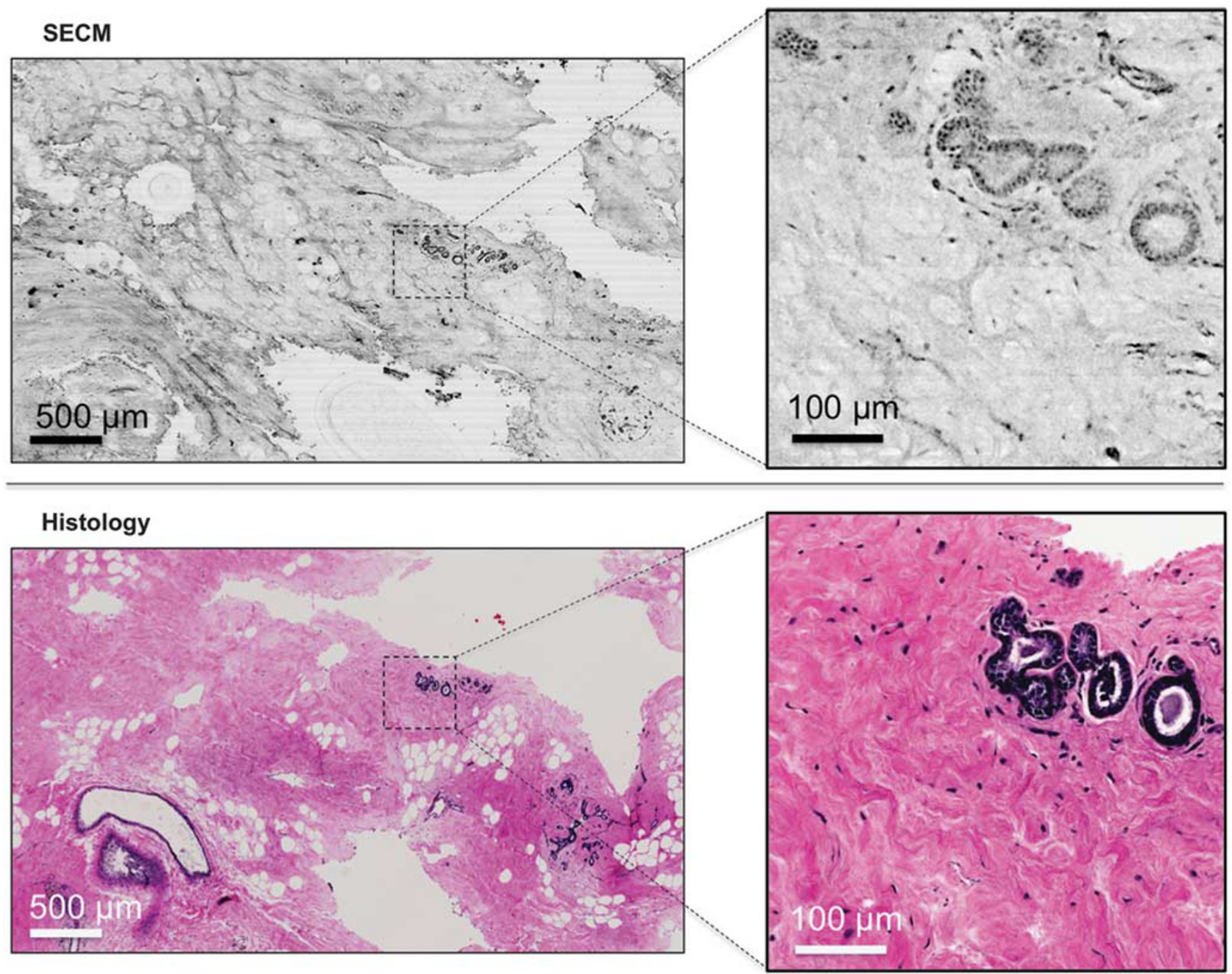

Figure 2 Representative spectrally encoded confocal microscopy (SECM) and histologic images of a breast tissue as an overview at low magnification (left) and in high magnification (right) with magnified area in box insert.

\section{SECM Image of Malignant Breast Tissue}

Representative SECM and histologic images of malignant breast tissues are shown in Figure 4. In both SECM and histologic images of DCIS (Figures 4a and b), a cribriform architecture (asterisks) is clearly shown and tumor cells are well contained inside the ductal membrane (dotted lines). In Figures $4 \mathrm{c}$ and $\mathrm{d}$, low-grade IDC is visualized with small irregularly shaped glands (dotted lines) invading the surrounding stroma. An SECM image of high-grade IDC (Figure 4e) enables the visualization of diffuse growth of tumor cells with virtually no stroma; comparable features are also seen in the corresponding histologic image (Figure 4f). In SECM and histologic images of ILC (Figures $4 \mathrm{~g}$ and $\mathrm{h}$ ), small and dyshesive tumor cells (arrows) are scattered between normal-looking stroma.

\section{Diagnostic Accuracy Evaluation}

Among the 124 pairs of SECM and histologic images used during diagnostic accuracy evaluation, 49 were benign/ normal and 75 were malignant per gold standard. Time required for rendering diagnoses on 124 images during each image review session was from 15 to 20 min for both SECM and histology.

Sensitivity and specificity evaluation results are summarized in Table 1. Sensitivity for SECM was high, ranging from 0.89 (95\% CI: $0.8-0.95$ ) to 0.95 (0.87-0.98), and average sensitivity was 0.91 . SECM specificity was also high, ranging from 0.88 $(0.76-0.94)$ to $0.98(0.89-1)$, and average value was 0.93 . Histology sensitivity ranged from $0.85(0.76-0.92)$ to $1(0.95-1)$ and specificity from $0.96(0.86-0.99)$ to $1(0.93-1)$. Average sensitivity and specificity for SECM were slightly lower than those for histology. There were statistically significant differences $(P<0.05)$ in two of the sensitivity measurements and two of the specificity measurements, highlighted in Table 1. The other four sensitivity measurements and four specificity measurements did not show statistically significant differences. Logistic regression showed that overall sensitivity and specificity of SECM were significantly different from 


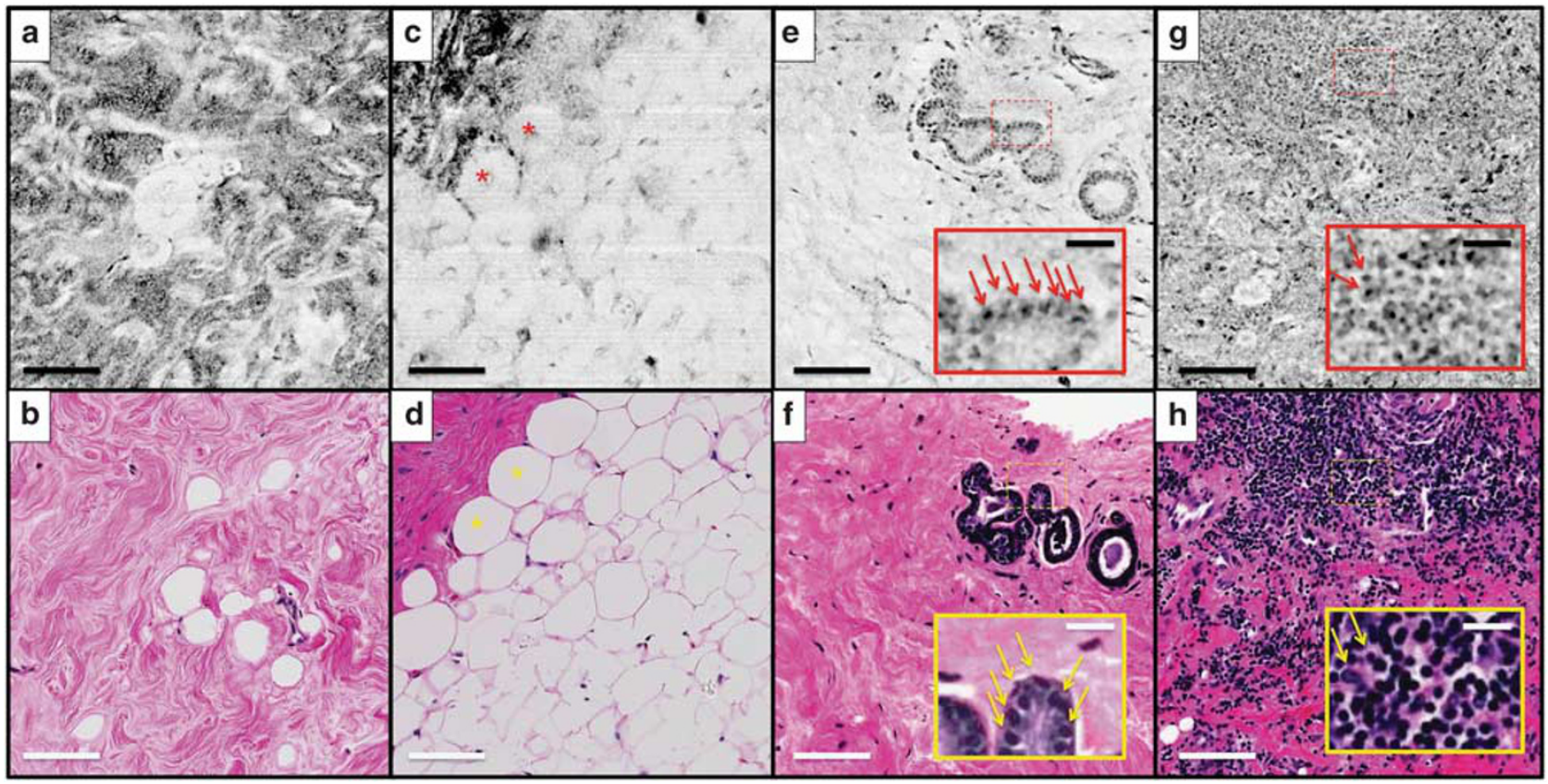

Figure 3 Representative spectrally encoded confocal microscopy (SECM) and histologic images of normal/benign breast tissues. (a, $\mathbf{c}$, e, and $\mathbf{g}$ ) SECM images. (b, d, f, and $\mathbf{h}$ ) Histologic images. (a and b) Fibrous tissue. (c and $\mathbf{d})$ Adipose tissue. (e and $\mathbf{f}$ ) Benign ducts and glands. (g and $\mathbf{h})$ Inflammation. Asterisks, fat cells; arrows, cell nuclei. Scale bars $=100 \mu \mathrm{m}$; scale bars in the insets $=20 \mu \mathrm{m}$.

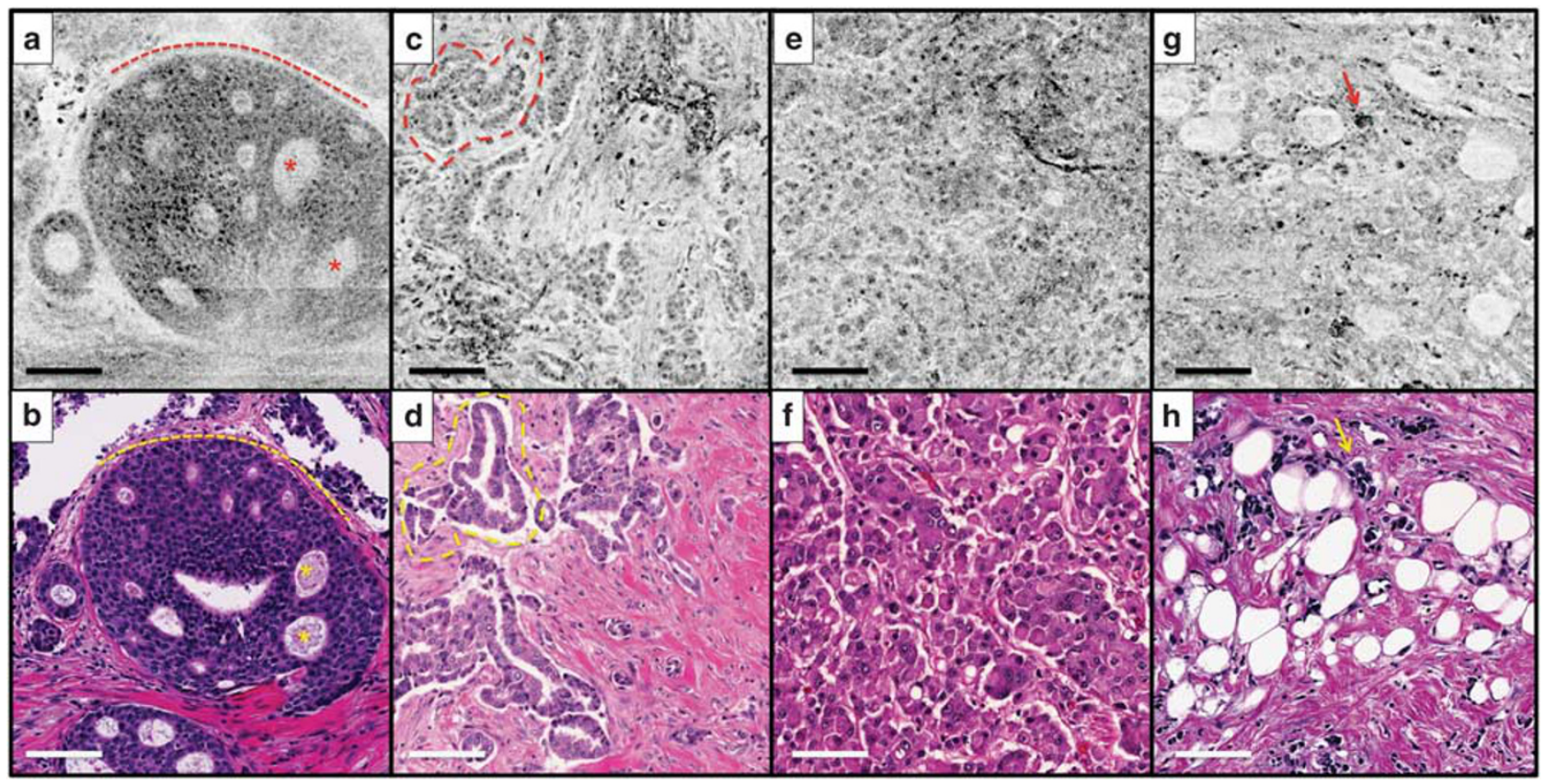

Figure 4 Representative spectrally encoded confocal microscopy (SECM) and histologic images of malignant breast tissues. (a, c, e, and $\mathbf{g})$ SECM images. (b, d, $\mathbf{f}$, and $\mathbf{h}$ ) Histologic images. (a and $\mathbf{b}$ ) Round foci of ductal carcinoma in situ (DCIS). (c and d) Low-grade invasive ductal carcinoma (IDC) with formation of irregular glandular structures. (e and $\mathbf{f}$ ) High-grade IDC with diffuse sheets of tumor cells. (g and $\mathbf{h}$ ) Invasive lobular carcinoma (ILC) with individual invasive cells. Asterisks, cribriform architecture; dotted line in $\mathbf{a}$ and $\mathbf{b}$, basement membrane; dotted line in $\mathbf{c}$ and $\mathbf{d}$, irregularly-shaped glands; arrows, tumor cells. Scale bars $=100 \mu \mathrm{m}$. 
Table 1 Sensitivity and specificity test results

\begin{tabular}{|c|c|c|c|c|c|c|}
\hline & \multicolumn{2}{|c|}{ SECM } & \multicolumn{2}{|c|}{ Histology } & \multicolumn{2}{|c|}{$P$-value } \\
\hline & Sensitivity & Specificity & Sensitivity & Specificity & Sensitivity & Specificity \\
\hline \multirow[t]{2}{*}{ Pathologist 1} & $0.91(0.82-0.95)$ & $0.96(0.86-0.99)$ & $1.00(0.95-1.00)$ & $0.96(0.86-0.99)$ & 0.01 & 1.00 \\
\hline & $0.89(0.80-0.95)$ & $0.92(0.81-0.97)$ & $0.99(0.93-1.00)$ & $0.96(0.86-0.99)$ & 0.01 & 0.41 \\
\hline \multirow[t]{2}{*}{ Pathologist 2} & $0.89(0.80-0.95)$ & $0.98(0.89-1.00)$ & $0.85(0.76-0.92)$ & $1.00(0.93-1.00)$ & 0.46 & 0.31 \\
\hline & $0.95(0.87-0.98)$ & $0.92(0.81-0.97)$ & $0.99(0.93-1.00)$ & $1.00(0.93-1.00)$ & 0.17 & 0.046 \\
\hline \multirow[t]{2}{*}{ Pathologist 3} & $0.91(0.82-0.95)$ & $0.92(0.81-0.97)$ & $0.96(0.89-0.99)$ & $1.00(0.93-1.00)$ & 0.17 & 0.046 \\
\hline & $0.92(0.84-0.96)$ & $0.88(0.76-0.94)$ & $0.95(0.87-0.98)$ & $0.98(0.89-1.00)$ & 0.51 & 0.06 \\
\hline Average & 0.91 & 0.93 & 0.96 & 0.98 & $<0.01$ & $<0.001$ \\
\hline
\end{tabular}

SECM, spectrally encoded confocal microscopy.

Numbers in parenthesis show lower and upper limits of $95 \%$ confidence interval. $P$-values $<0.05$ are highlighted by italic fonts.

Table 2 Intra-observer agreement evaluation results

\begin{tabular}{lccc}
\hline & SECM & Histology & $P$-value \\
\hline Pathologist 1 & $0.88(0.80-0.97)$ & $0.98(0.95-1.00)$ & 0.03 \\
Pathologist 2 & $0.82(0.72-0.92)$ & $0.84(0.74-0.93)$ & 0.79 \\
Pathologist 3 & $0.92(0.84-0.99)$ & $0.93(0.87-1.00)$ & 0.72 \\
Average & 0.87 & 0.92 & \\
& & &
\end{tabular}

SECM, spectrally encoded confocal microscopy.

Numbers in parenthesis show lower and upper limits of $95 \%$ confidence interval. $P$-values $<0.05$ are highlighted by italic fonts.

those of histology, $P$-values $<0.01$ and 0.001 , respectively. Average PPV for SECM was 0.95 and NPV was 0.87. Average PPV and NPV for histology were 0.99 and 0.94 , respectively. Average diagnostic accuracy for SECM was 0.92 and average accuracy for histology was 0.97 .

Intra-observer agreement evaluation results are summarized in Table 2. Intra-observer agreement for SECM was high, ranging from $0.82(0.72-0.92)$ to $0.92(0.84-0.99)$, and average $\kappa$-value was 0.87 . Similarly, intra-observer agreement for histology was high, ranging from $0.84(0.74-0.93)$ to 0.98 $(0.95-1)$, with average of 0.92 . One of three $\kappa$-value comparisons showed a statistically significant difference.

Table 3 shows summary of inter-observer agreement evaluation results. Inter-observer agreement for SECM diagnoses was high, ranging from $0.77(0.66-0.88)$ to 0.87 $(0.78-0.96)$, with an average of 0.84 . Histology also showed high inter-observer agreement, ranging from $0.79(0.68-0.90)$ to $0.97(0.92-1.00)$, with an average of 0.90 . There was one $\kappa$ value comparison that showed a statistically significant difference between SECM and histology.

\section{Discussion on Discrepant Cases}

There were four cases where more than half of the six SECM diagnoses were incorrect, whereas more than half of the six
Table 3 Inter-observer agreement evaluation results

\begin{tabular}{lccc}
\hline & SECM & Histology & $P$-value \\
\hline Pathologist 1 vs 2 & $0.80(0.70-0.91)$ & $0.79(0.68-0.90)$ & 0.84 \\
& $0.83(0.74-0.93)$ & $0.97(0.92-1.00)$ & 0.02 \\
Pathologist 2 vs 3 & $0.77(0.66-0.88)$ & $0.87(0.78-0.96)$ & 0.17 \\
& $0.87(0.78-0.96)$ & $0.93(0.87-1.00)$ & 0.23 \\
Pathologist 3 vs 1 & $0.87(0.78-0.96)$ & $0.92(0.84-0.99)$ & 0.41 \\
& $0.87(0.78-0.96)$ & $0.93(0.87-1.00)$ & 0.24 \\
Average & 0.84 & 0.90 & \\
\hline
\end{tabular}

SECM, spectrally encoded confocal microscopy.

Numbers in parenthesis show lower and upper limits of $95 \%$ confidence interval. $P$-values $<0.05$ are highlighted by italic fonts.

histologic diagnoses were correct. Two of these cases were SECM malignant and histology normal/benign, and showed similar histomorphologic features. One of these cases is shown in Figures $5 \mathrm{a}$ and $\mathrm{b}$. The gold standard diagnosis was ducts/glands. In SECM image (Figure 5a), there are scattered areas where cell nuclei are not reliably visualized, which made it challenging to delineate boundary of each acinus and made the SECM image appear similar to IC with no stroma. The other two cases were SECM normal/benign and histology malignant, and exhibited similar microscopic features. Figures $5 c$ and $d$ show SECM and histologic images for one of these cases. The gold standard diagnosis for these images was ILC. SECM image (Figure 5c) appears to have poor resolution: cell nuclei in the stroma are not well visualized (dotted line) and boundaries of fat cells are not clearly distinguished (right side and bottom).

\section{DISCUSSION}

Results from this study show that sensitivity and specificity of SECM for detecting malignant breast tumors are high. SECM 


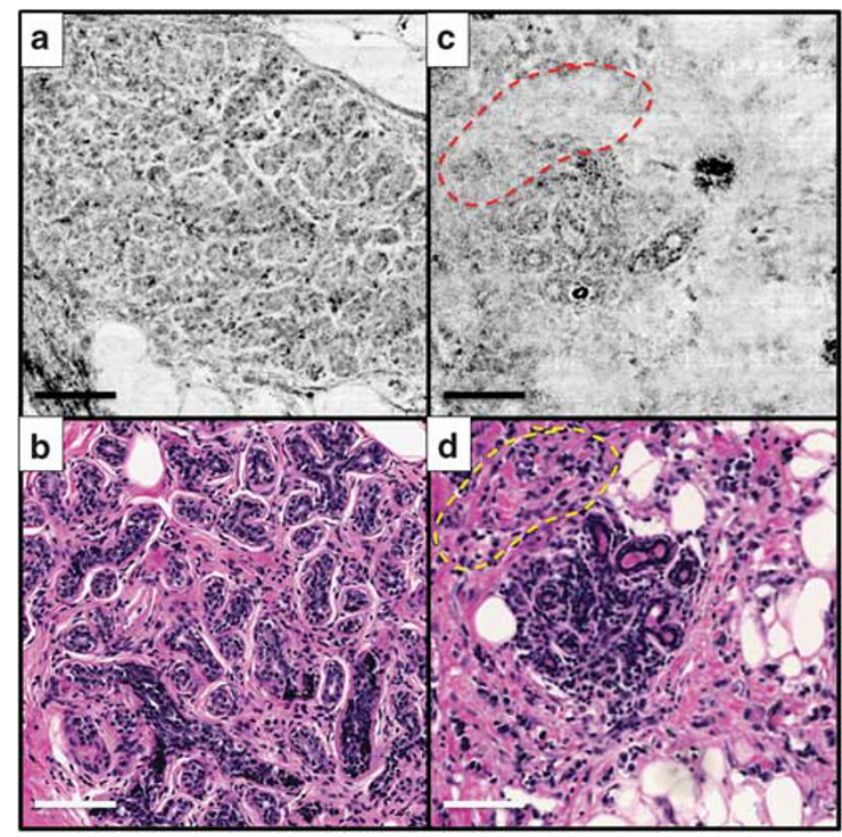

Figure 5 Representative spectrally encoded confocal microscopy (SECM) and histologic images for cases with incorrect SECM diagnoses but correct histologic diagnoses. (a and b) Ducts/glands. (c and d) Invasive lobular carcinoma (ILC). Dotted line, area where SECM image failed to clearly visualize cell nuclei in stroma. Scale bars $=100 \mu \mathrm{m}$.

sensitivity and specificity are similar to the sensitivity and specificity previously reported for other optical microscopy technologies. ${ }^{19,20}$ Study results also show that intra- and inter-observer agreements for SECM are high. With the high diagnostic accuracy demonstrated in this study and fast imaging speed shown in our previous work, ${ }^{24}$ SECM will uniquely enable microscopic imaging of the entire breast excision specimens in a short imaging time and provide accurate margin status during the surgery. Real-time feedback regarding the margin status will be used to conduct additional re-resections to finally achieve negative margin during the initial surgery, which can obviate the need for additional surgeries.

Average SECM sensitivity and specificity were lower than average histologic sensitivity and specificity. SECM occasionally produced poor image quality for a few images (Figures $5 \mathrm{a}$ and c). If we exclude the four poor-image-quality cases described in the Results section, average SECM sensitivity and specificity become 0.94 and 0.97 , respectively, and differences in average sensitivity and specificity between SECM and histology become statistically insignificant $(P$-values $=0.10$ and 0.12 , respectively). SECM images obtained from superficial regions visualized cellular features in a similar manner to histologic images even though the lateral resolution was worse for SECM than the whole slide scanner, $1.3 \mu \mathrm{m}$ versus $0.43 \mu \mathrm{m}$. At large imaging depths, the effective resolution of SECM was significantly degraded due to light scattering and optical aberrations, which resulted in poor image quality as exemplified in Figures $5 \mathrm{a}$ and c. This study has been helpful in educating us about the difficulties of diagnosing SECM images that are too deep below the surface of the tissue. These findings suggest that during intra-operative imaging, SECM images should be obtained from a superficial focal plane, where resolution is retained and diagnostic accuracy is likely to be higher.

There were several limitations in this study: (1) diagnostic accuracy was tested by reviewing small images. Accuracy of determining margin status by reviewing a large SECM image of the entire surgical specimen still needs to be evaluated. (2) Similarly, the amount of time needed to review a large SECM image at various magnifications and determine margin status needs to be studied. (3) Breast samples were resected by scalpels from mastectomy or excision specimens and did not have cautery artifacts. Effects of cautery artifacts on SECM diagnostic accuracy are not known yet. (4) Histologic margin assessment is generally conducted on cross-sections of the tissue, whereas SECM margin assessment is conducted on en face planes. Clinical implication of the en face SECM margin status has not been studied. (5) Margin width information is not obtained in SECM due to en face manner of SECM imaging and its limited imaging depth, typically around $200 \mu \mathrm{m}$. Recent guideline on margins for breast-conserving surgery found that although a negative margin lowers risk of local recurrence, there is no statistically significant correlation between margin width and reduction of local recurrence rate. ${ }^{1}$ We therefore expect that lack of margin width information will not limit SECM's capability to guiding breast cancer excision. This hypothesis, however, will need to be tested in future clinical studies. (6) Study samples mainly included common breast cancers but did not have more complex diseases, including atypical intraductal proliferations, small foci of carcinoma, lymphatic vessel invasion, and microinvasive carcinoma in a background of extensive DCIS. Diagnostic accuracies for these complex diseases need to be evaluated.

SECM still needs technical improvements on the following items before it can be fully used for clinical intraoperative applications: (1) Although the fast SECM imaging speed $\left(100 \mathrm{kHz}\right.$ line rate $\left.{ }^{24}\right)$ will enable imaging of the entire excision specimen (typical size $=\sim 30 \mathrm{~cm}^{2}$ ) in a short time $(7.5 \mathrm{~min}$ ), faster imaging speed might be needed to allow for more time to review SECM images. We can increase the imaging speed further by using a higher-rate swept source (several MHz) 27,28 and higher speed detectors and digitizer systems. (2) Adequate software needs to be developed for fast image display and navigation, to enable intraoperative margin assessment with SECM images. We will develop a fast image display/navigation method that are similar to those used in whole slide imaging (WSI) systems, where original large image with full resolution is converted into a pyramidal structure for rapid image display/navigation. With an adequate image display/navigation software, we expect that time from tissue excision to final SECM margin determination will be similar to turn around time for WSI-based 
diagnosis of a frozen section, around $15 \mathrm{~min},{ }^{29}$ as image review time was found to be similar between SECM and histology in this study. (3) An automated tissue type recognition software could be used to further reduce the image review time. The automated software could identify regions that need to be examined by the pathologist. By focusing on these selected regions, the pathologist could complete the image review within a shorter time. Previously, several algorithms were developed to automatically analyze histomorphologic patterns in histologic images of breast tissues. ${ }^{30,31}$ We will develop a similar pattern analysis algorithm for SECM breast images. (4) A systematic scanning method needs to be developed, to image the entire surface of the breast excision specimen. Although development of the scanning method will need to be done in conjunction with end user surgeon and pathologist requirements, at present we suggest the following protocol: (i) for shaved margin specimens, when oriented, the patient-side surface will be comprehensively imaged, whereas if not oriented, both sides will be imaged; (ii) for lumpectomy specimens, the SECM device will be configured to scan in three dimensions over the surface of the specimen and registered with orientation landmarks provided by the surgeon.

In the future, we will develop an intraoperative SECM system that can be used to study and address the aforementioned technical challenges. We will then conduct a clinical study of using the intraoperative SECM system in guiding breast cancer excision. In this future study, we will image the entire excision specimens with SECM, determine margin status, and resect additional tissues based on the SECM margin status along with findings from other intraoperative imaging modalities. From this clinical study, we will be able to evaluate effectiveness of SECM for reducing the need for additional surgeries.

\section{ACKNOWLEDGMENTS}

We thank Jeonghyun Lee for his help on digitizing histology slides. We also thank the MGH surgical pathology unit for their help on procuring surgical breast tissues. This work was supported by Center for Integration of Medicine and Innovative Technology (CIMIT) under US Army Medical Research Acquisition Activity Cooperative Agreement (W81XWH-09-2-0001). This work was conducted with support from Harvard Catalyst | The Harvard Clinical and Translational Science Center (National Center for Research Resources and the National Center for Advancing Translational Sciences, National Institutes of Health Award UL1 TR001102) and financial contributions from Harvard University and its affiliated academic healthcare centers. The information contained herein does not necessarily reflect the position or policy of the Government, and no official endorsement should be inferred. The content is solely the responsibility of the authors and does not necessarily represent the official views of Harvard Catalyst, Harvard University and its affiliated academic healthcare centers, or the National Institutes of Health.

\section{DISCLOSURE/CONFLICT OF INTEREST}

AEH is currently with Saint Barnabas Medical Center (Livingston, NJ). GJT and DK are inventors of patents and patent applications on the spectrally encoded confocal microscopy technology described in this study. These patents and patent applications are not currently licensed. All other authors declare no conflict of interest.
1. Moran MS, Schnitt SJ, Giuliano AE et al. Society of Surgical OncologyAmerican Society for Radiation Oncology consensus guideline on margins for breast-conserving surgery with whole-breast irradiation in stages I and II invasive breast cancer. Int J Radiat Oncol Biol Phys 2014;88:553-564.

2. McCahill LE, Single RM, Aiello Bowles EJ et al. Variability in reexcision following breast conservation surgery. JAMA 2012;307:467-475.

3. Morrow M, Jagsi R, Alderman AK et al. Surgeon recommendations and receipt of mastectomy for treatment of breast cancer. JAMA 2009;302: 1551-1556.

4. Coopey SB, Buckley JM, Smith BL et al. Lumpectomy cavity shaved margins do not impact re-excision rates in breast cancer patients. Ann Surg Oncol 2011;18:3036-3040.

5. Boughey JC, Hieken TJ, Jakub JW et al. Impact of analysis of frozensection margin on reoperation rates in women undergoing lumpectomy for breast cancer: evaluation of the National Surgical Quality Improvement Program data. Surgery 2014;156:190-197.

6. Uecker JM, Bui EH, Foulkrod KH et al. Intraoperative assessment of breast cancer specimens decreases cost and number of reoperations. Am Surg 2011;77:342-344.

7. Esbona K, Li Z, Wilke LG. Intraoperative imprint cytology and frozen section pathology for margin assessment in breast conservation surgery: a systematic review. Ann Surg Oncol 2012;19:3236-3245.

8. D'Halluin F, Tas P, Rouquette $S$ et al. Intra-operative touch preparation cytology following lumpectomy for breast cancer: a series of 400 procedures. Breast 2009;18:248-253.

9. Sharma V, Shivalingaiah S, Peng Y et al. Auto-fluorescence lifetime and light reflectance spectroscopy for breast cancer diagnosis: potential tools for intraoperative margin detection. Biomed Opt Express 2012;3: 1825-1840.

10. Keller MD, Majumder SK, Kelley MC et al. Autofluorescence and diffuse reflectance spectroscopy and spectral imaging for breast surgical margin analysis. Lasers Surg Med 2010;42:15-23.

11. Nachabé R, Evers DJ, Hendriks $\mathrm{BH}$ et al. Diagnosis of breast cancer using diffuse optical spectroscopy from 500 to $1600 \mathrm{~nm}$ : comparison of classification methods. J Biomed Opt 2011;16:087010.

12. Brown JQ, Bydlon TM, Kennedy SA et al. Optical spectral surveillance of breast tissue landscapes for detection of residual disease in breast tumor margins. PLoS One 2013;8:e69906.

13. Laughney AM, Krishnaswamy V, Rizzo EJ et al. Scatter spectroscopic imaging distinguishes between breast pathologies in tissues relevant to surgical margin assessment. Clin Cancer Res 2012;18: 6315-6325.

14. Lue N, Kang JW, Yu CC et al. Portable optical fiber probe-based spectroscopic scanner for rapid cancer diagnosis: a new tool for intraoperative margin assessment. PLoS One 2012;7:e30887.

15. Keller MD, Vargis E, de Matos Granja N et al. Development of a spatially offset Raman spectroscopy probe for breast tumor surgical margin evaluation. J Biomed Opt 2011;16:077006.

16. Haka AS, Volynskaya Z, Gardecki JA et al. In vivo margin assessment during partial mastectomy breast surgery using Raman spectroscopy. Cancer Res 2006;66:3317-3322.

17. Nguyen FT, Zysk AM, Chaney EJ et al. Intraoperative evaluation of breast tumor margins with optical coherence tomography. Cancer Res 2009;69:8790-8796.

18. Assayag O, Antoine M, Sigal-Zafrani B et al. Large field, high resolution full-field optical coherence tomography: a pre-clinical study of human breast tissue and cancer assessment. Technol Cancer Res Treat 2014;13:455-468.

19. Dobbs JL, Ding H, Benveniste AP et al. Feasibility of confocal fluorescence microscopy for real-time evaluation of neoplasia in fresh human breast tissue. J Biomed Opt 2013;18:106016.

20. Tao YK, Shen D, Sheikine $Y$ et al. Assessment of breast pathologies using nonlinear microscopy. Proc Natl Acad Sci USA 2014;111: 15304-15309.

21. Schiffhauer LM, Boger JN, Bonfiglio TA et al. Confocal microscopy of unfixed breast needle core biopsies: a comparison to fixed and stained sections. BMC Cancer 2009;9:265.

22. Tilli MT, Cabrera MC, Parrish AR et al. Real-time imaging and characterization of human breast tissue by reflectance confocal microscopy. J Biomed Opt 2007;12:051901.

23. Tearney GJ, Webb RH, Bouma BE. Spectrally encoded confocal microscopy. Opt Lett 1998;23:1152-1154. 
24. Schlachter SC, Kang D, Gora MJ et al. Spectrally encoded confocal microscopy of esophageal tissues at $100 \mathrm{kHz}$ line rate. Biomed Opt Express 2013;4:1636-1645.

25. Kang D, Suter MJ, Boudoux $C$ et al. Comprehensive imaging of gastroesophageal biopsy samples by spectrally encoded confocal microscopy. Gastrointest Endosc 2010;71:35-43.

26. Kang DK, Suter MJ, Boudoux C et al. Co-registered spectrally encoded confocal microscopy and optical frequency domain imaging system. J Microsc 2010;239:87-91.

27. Reznicek L, Klein T, Wieser W et al. Megahertz ultra-wide-field sweptsource retina optical coherence tomography compared to current existing imaging devices. Graefes Arch Clin Exp Ophthalmol 2014;252: 1009-1016.
28. Xu J, Zhang C, Xu J et al. Megahertz all-optical swept-source optical coherence tomography based on broadband amplified optical timestretch. Opt Lett 2014;39:622-625.

29. Pantanowitz L, Valenstein PN, Evans AJ et al. Review of the current state of whole slide imaging in pathology. J Pathol Inform 2011;2:36

30. Naik $S$, Doyle $S$, Agner $S$ et al. Automated gland and nuclei segmentation for grading of prostate and breast cancer histopathology. Proceedings of the Biomedical Imaging: From Nano to Macro, 2008. ISBI 2008. 5th IEEE International Symposium on; 2008; IEEE.

31. Petushi S, Garcia FU, Haber MM et al. Large-scale computations on histology images reveal grade-differentiating parameters for breast cancer. BMC Med Imaging 2006;6:14. 